\title{
PROBLEM OF ORBITAL IMPLANTS AFTER ENUCLEATION*†
}

BY

\section{H. ARRUGA}

Barcelona

GREAT efforts have been made by ophthalmologists in the last 10 years to design an implant conveying good prosthetic mobility, to offset in part the tragic result of excision. The immediate success of the implants aroused enthusiasm, but subsequent complications, including suppuration and expulsion, have led to further modifications. So frequent were these disappointments in spite of changes in technique that most ophthalmologists returned to the idea of a less mobile implant, which would neither cause suppuration nor be expelled.

It became evident that these complications were due to the fact that where part of an implant protruded through an open wound, the wound rarely healed. As I had the same disappointments as others with this technique, I used a three-quarter sphere plastic implant with tunnels through which the four recti were drawn and sutured. This implant was completely buried and had no peg attached to it.

My friend Moura-Brazil had observed that if an implant had a peg buried some distance from the conjunctival wound, it would slowly perforate the conjunctiva without any visible wound being formed around it. Since this implant had certain defects of shape in my opinion, he and I agreed to add a peg to my implant, to employ his method of conjunctival suture and to name this the Arruga-Moura-Brazil technique. The result is an acrylic implant $3 / 4$ or $2 / 5$ of a sphere of $16-18 \mathrm{~mm}$. diameter with one or two noncorrodible metal pegs $1 \mathrm{~mm}$. thick and $7 \mathrm{~mm}$. long projecting from the upper part of its anterior surface (Figs 1 and 2).

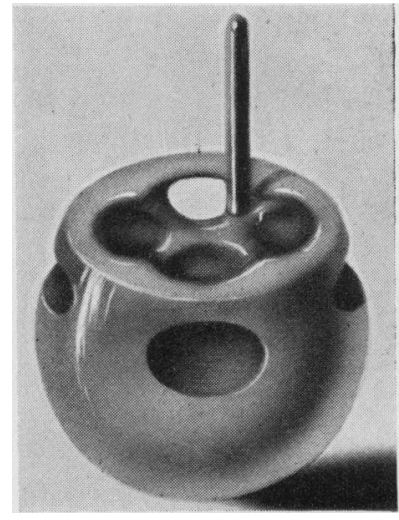

FIG. 1.-Implant Type I with one peg.

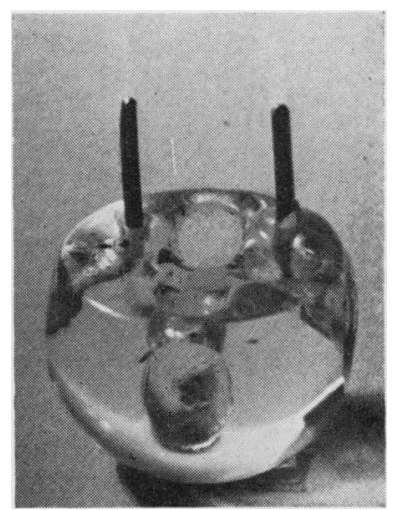

FIG. 2.-Implant Type I with two pegs.

*Received for publication May 20, 1955.

† Montgomery Lecture, Dublin, 1955. 


\section{Operation I}

The conjunctiva and episclera are dissected posteriorly as far as the insertions of the recti tendons. A suture is passed through each tendon which are then divided close to their insertions. The eye is excised using Foster's snare to diminish haemorrhage. The implant is placed with two pegs protruding forward from its upper surface. The tendons of the vertical recti muscles are passed through the tunnels of the implant and sutured together; the tendons of the horizontal recti are treated similarly. An additional suture is passed through all four tendons as a precaution in case one of the other sutures slips. Sulphonamide or penicillin powder may be inserted into the wound and the conjunctiva and Tenon's capsule sutured in such a way that the pegs are buried, but not included in the wound.

The conjunctival sutures are removed a week later. The pegs slowly perforate the overlying tissues in 2 to 4 weeks. Should this be delayed, a small cut over the tissues on top of the peg will complete the process (Figs 3-8, opposite).

The post-operative course is generally uneventful and a provisional prosthesis that need not be provided with holes for the pegs can be fitted, since its role is to prevent the retraction of the fornices.

Prosthesis.-A semi-final acrylic prosthesis can be fitted 2 or 3 months after the operation-I say " semi-final " because in most cases the orbital contents retract and a new prosthesis has to be made. In some cases the position of the pegs alters so that a new prosthesis is required or new holes must be drilled on the back of the old one.

The mobility of the prosthesis is generally very satisfactory, although it varies in each case; movements are generally 50-70 per cent. of the normal horizontally and $40-60$ per cent. vertically.

The problem whether the implant should have one or two pegs is a matter of opinion. The single peg has the disadvantage that the prosthesis may rotate around it. To avoid this rotation, a single-peg prosthesis must be provided with a prominence which enters the fornix and thus limits movement in that direction. The prosthesis is usually made with the prominence upwards, since in this direction movement is least important; moreover, it largely eliminates the superior palpebral furrow which is an almost constant complication of prostheses after some years (Fig. 9, overleaf). If the twin-peg implant is used this prominence is unnecessary; mobility is then greater (Fig. 10, overleaf) but the retraction into the upper lid still occurs.

Results.-Since 1951, when I started to use the technique described, I have performed 39 operations, using implants with either one or two pegs. The immediate results are almost constantly excellent, but complications may sometimes occur that make a further operation necessary. The complications I have observed are as follows:

(l) A 10-year-old girl.-14 months after operation with a perfect result, the prosthesis suddenly turned upwards. An oculist operated and found the inferior rectus detached and inaccessible, and 2 months later the same thing happened to the external rectus. I therefore removed the implant and inserted a buried plastic sphere.

(2) A 24-year-old man.-After he had worn the prosthesis for 2 years, sudden exophthalmos occurred with pain and fever, but no manifest conjunctival suppuration. Conjunctival chemosis made the prosthesis unwearable. Sulphonamides, penicillin, and streptomycin brought about recovery from the inflammation in 6 days. He has now worn the prosthesis for 3 months with no trouble. 


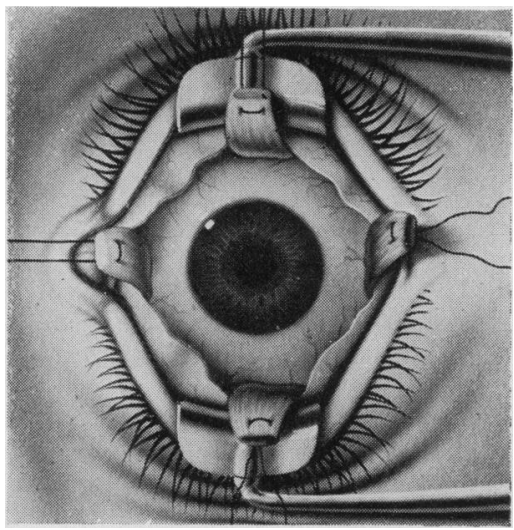

FIG. 3.-I (1) Disinsertion of four recti; a thread passed through each tendon.

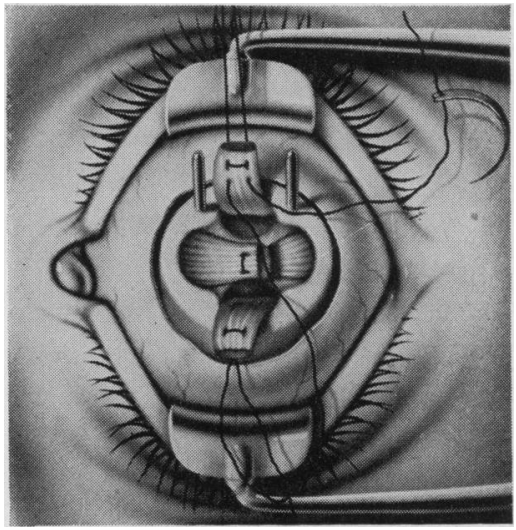

FIG. 5.--I (3) Superior and inferior recti passed through holes of the implant.

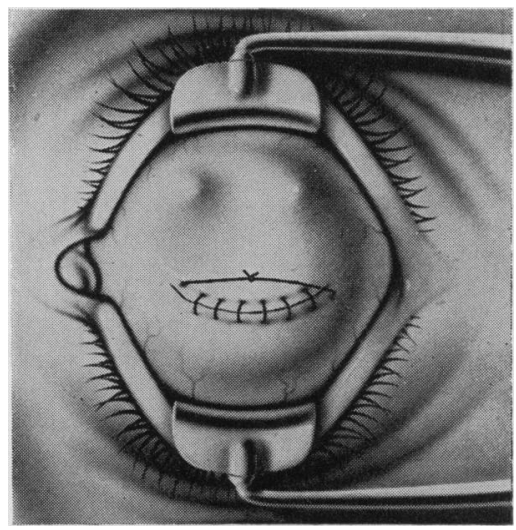

FIG. 7.-I (6) Suture of conjunctiva. The pegs are covered by the conjunctiva.

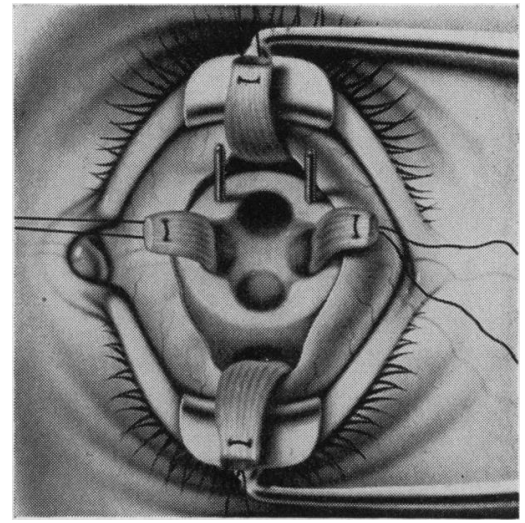

FIG. 4.-I (2) Internal and external recti passed through holes of the implant.

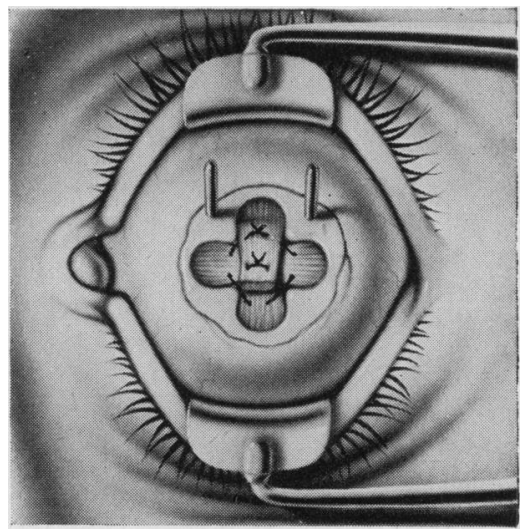

FIG. 6.-I (5) Threads knotted and suture passed through all four tendons.

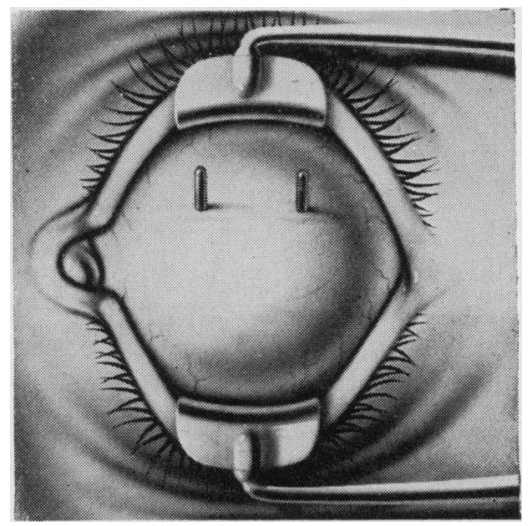

FIG. 8.-I (7) After a few days the pegs perforate the conjunctiva. 

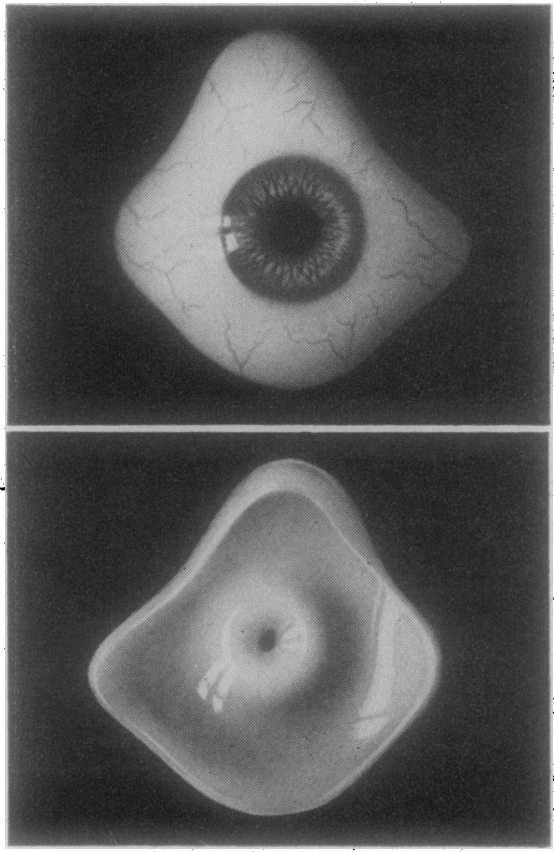

FIG. 9.-Artificial eye for one peg with prominence upwards.
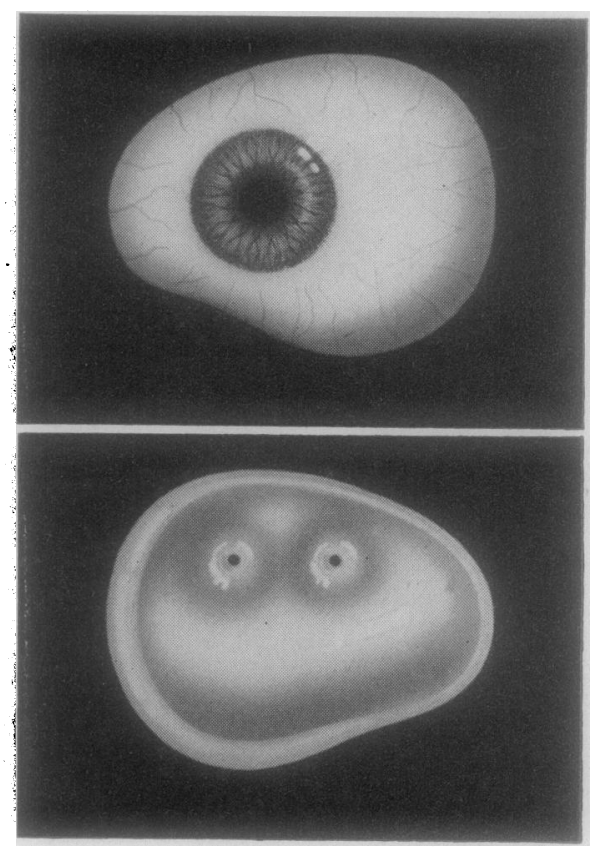

FIG. 10.-Artificial eye for two pegs.

(3) A 26-year-old woman.-Operation with immediate good result despite intense bleeding. The wound gradually enlarged, reaching the base of the peg 2 months later. Intense and obstinate suppuration obliged me to remove the implant.

(4) A 43-year-old male. - The operation was difficult because old inflammation had left a thin and friable conjunctiva and episclera. At the end of the operation the peg was visible through the tissues, and 2 weeks later the conjunctiva perforated and the wound became progressively larger. I then covered the implant with mucous membrane from the lip with a good result which remained satisfactory for 6 months when the patient was last seen; since then I understand this state of affairs has continued.

(5) A 27-year-old male.-The operation was followed by an excellent result, but 8 months later the prosthesis slowly deviated upwards and outwards. The patient's own oculist observed that, despite this, the mobility was good in all directions. Drilling another hole in the back of the artificial eye rendered the appearance satisfactory, and it remained so 3 months later. Possibly a partial disinsertion occurred, but nearby adhesions preserved the action of the muscle.

The other cases were uncomplicated, apart from discharge, but this is no greater than is produced by an artificial eye without an implant. No treatment is needed except boric lotion twice a day.

Consideration of the complicated cases has led me to wonder whether, when disinsertion of the tendons occurs late (in one of my cases 14 months after operation), it may be due to necrosis of the tendons from the tightness of the sutures, or to a low grade infection around them. In view of this, I have devised a new technique, leaving a scleral ring to which the four recti remain inserted. 


\section{Modified Technique}

Operation II.-The conjunctiva is dissected from the limbus almost to the equator and the cornea is excised close to the limbus. The contents of the eye are removed as in an exenteration. There is no need to stress the "cleaning" of the posterior hemisphere of the sclera, since this is excised.

Through the corneal opening, the sclera is seized with firm-toothed forceps at about $10 \mathrm{~mm}$. from the limbus, and the sclera is cut all round so that a scleral ring containing the insertions of the four rectus muscles is left extending from the limbus to the proximity of the equator. The posterior portion of the sclera is then pulled outwards, and the obliques and the optic nerve are cut (Figs 11-14).

The implant is introduced between the lateral and inferior recti so that it fits into the scleral ring from behind. Two small holes are pierced with a cataract knife in the borders of the limbus. Through these, the two pegs are passed, the conjunctiva and Tenon's capsule are sutured, and these tissues cover the pegs. The stitches are removed a week later (Figs 15-18, overleaf).

Implant.-The acrylic implant for this operation has a different shape, namely that of $3 / 4$ of a sphere of $18 \mathrm{~mm}$. diameter (Fig. 19, overleaf). The edge is smooth and the two pegs are about $10 \mathrm{~mm}$. apart, their length being $7 \mathrm{~mm}$. The advantage of this type is that it avoids leaving buried sutures and that there are no corners where blood could collect and become a focus of infection.

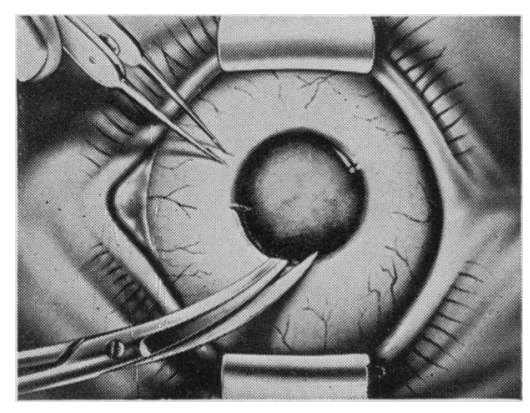

FIG. 11.-II (1) Excision of cornea.

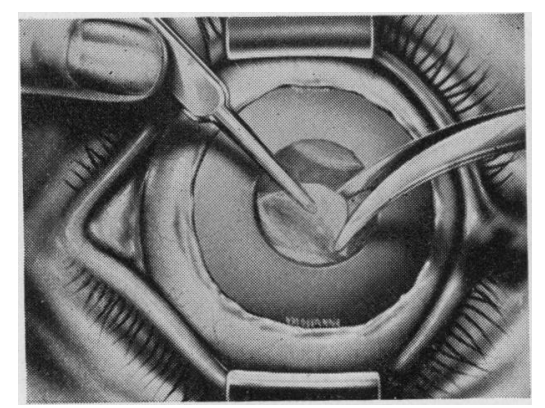

Fig. 13.-II (3) The sclera is cut all round at about $10 \mathrm{~mm}$. from the limbus.

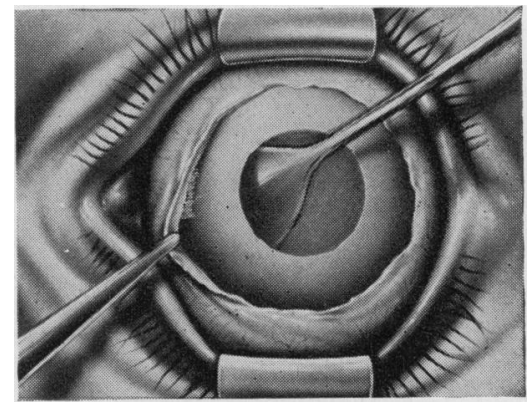

FIG. 12.-II (2) Eye contents removed.

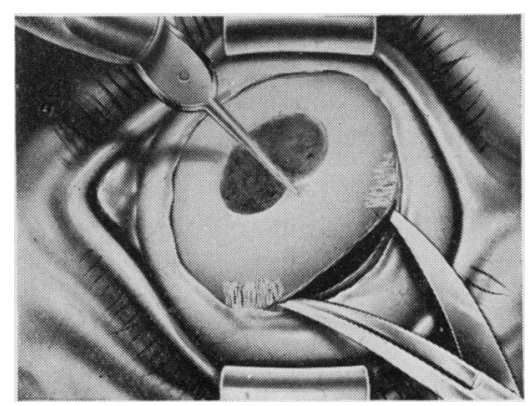

FIG. 14.-II (4) The space between the muscles is enlarged with the scissors. 


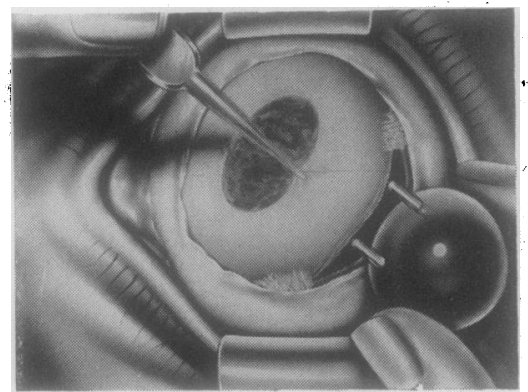

FIG. 15.-II (5) The implant is introduced between the lateral and inferior recti.

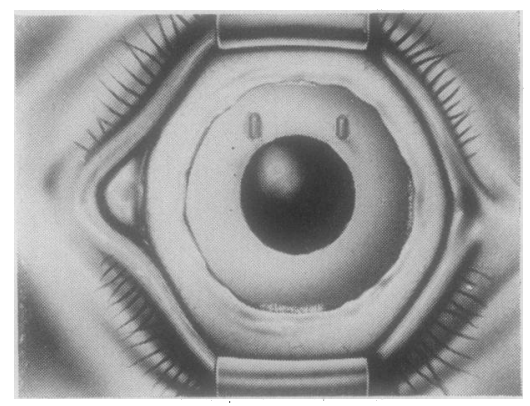

FIG. 17.-II (7) The pegs are passed through the holes.

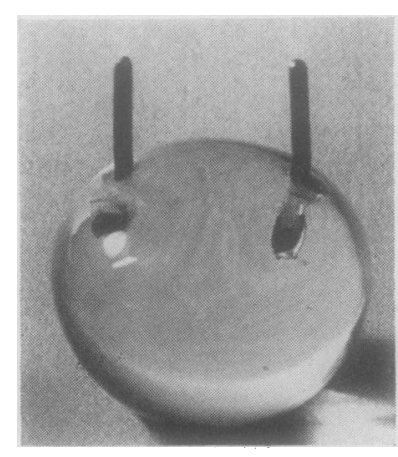

Fig. 19.--Implant Type II.

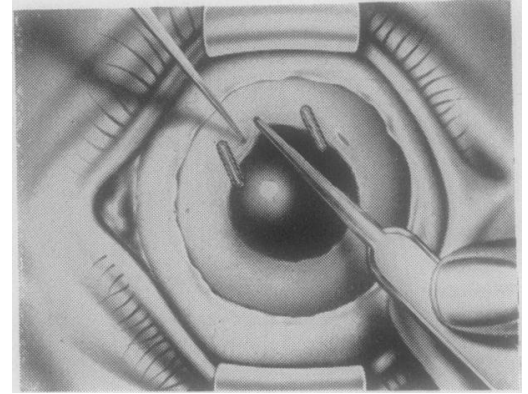

FIG. 16.-II (6) Two small holes are pierced in the borders of the sclera.

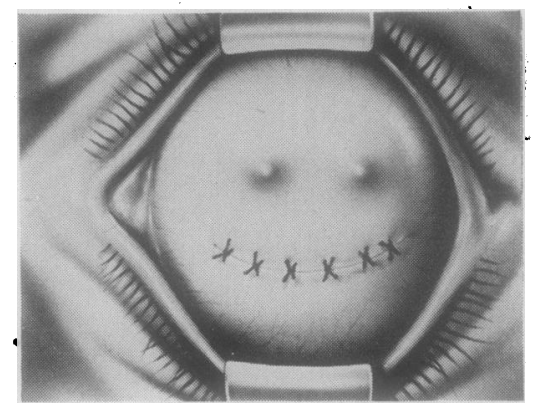

FIG. 18.--II (8) Suture of conjunctiva and Tenon's capsule. Pegs covered.

This second method has the disadvantage that it cannot be applied in cases of sarcoma of the choroid since handling of the tumour might cause local implantation or metastasis.

I have used this technique in five cases and, although the results are so far excellent, we must wait at least 2 years before we can be sure that it is an improvement on the other. It has given me great pleasure, however, to describe this method for the first time to the Irish Ophthalmological Society, who have so greatly honoured me by their invitation to deliver the Montgomery Lecture. 